\title{
Estimativa da evapotranspiração de referência no município de Capim, PB $^{1}$
}

\author{
Ednaldo A. Mendonça ${ }^{2}$ \& Renilson T. Dantas ${ }^{2}$
}

\section{RESUMO}

Objetivou-se, com este trabalho, estimar a evapotranspiração de referência diária para o município de Capim, PB, através dos métodos de Penman-Monteith, Linacre e Radiação, posteriormente correlacionados com a estação do ano, razão por que se coletaram dados meteorológicos da estação meteorológica automática, instalada na Fazenda Capim II, no período de 21 de dezembro de 2006 a 20 de dezembro de 2007, os quais foram registrados em intervalo de $3 \mathrm{~h}$. Verificou-se que, das correlações obtidas com o método de Penman-Monteith, se destacou a do método de Radiação, cujos coeficientes de determinação foram superiores a 0,80 .

Palavras-chave: agricultura, água, radiação, temperatura do ar

\section{Estimate of the reference evapotranspiration in the municipality of Capim, PB - Brazil}

\begin{abstract}
This study aimed to estimate the daily reference evapotranspiration for the town of Capim, PB, using the methods of Penman-Monteith, Linacre and Radiation, subsequently correlated with the season. For this, meteorological data were collected from the automatic weather station installed at Capim II Farm during the period of December 21, 2006 to December 20, 2007, recorded at intervals of $3 \mathrm{~h}$. From the correlations obtained with the method of Penman-Monteith, the radiation method stood out with the coefficients of determination greater than 0.80 .
\end{abstract}

Key words: agriculture, water, radiation, air temperature

1 Parte da Dissertação de Mestrado do primeiro autor

2 DCA/UFCG, Av. Aprígio Veloso 882, Bodocongó, CEP 58109-970, Campina Grande, PB. Fone: (83) 3310-1202. E-mail: professorednaldo@terra.com.br; renilson@dca.ufcg.edu.br 


\section{INTRODUÇÃO}

Os recursos hídricos de uma região devem ser utilizados de forma racional por toda a sociedade, uma vez que a água é a substância mais importante para a sobrevivência das espécies animal e vegetal, ressaltando-se que a necessidade do controle das reservas hídricas é fundamental para a existência de vida no planeta. Um dos setores de maior consumo mundial de água é a agricultura, daí a preocupação de se fazer cada vez mais o uso racional desse valioso líquido.

Para se alcançar economia hídrica na agricultura é oportuno se obter uma irrigação mais próxima do ideal e, para tanto, necessita-se de estudos que levem em consideração principalmente os aspectos meteorológicos da região mas, também, se fazer estimativas mais confiáveis para o manejo dessa técnica, em cujo contexto a estimativa adequada da evapotranspiração de referência (ETo) é de suma importância.

A água pode retornar à atmosfera através de dois processos naturais: por intermédio de evaporação e transpiração. De acordo com Turco et al. (2005), a evapotranspiração pode ser definida como um processo combinado de transferência de água do solo para a atmosfera, incluindo o processo de transpiração por meio dos tecidos vegetais.

Pozzebon et al. (2003) expressam, por outro lado, a evapotranspiração de referência (ETo) como sendo a água evaporada do solo e transpirada pelas plantas em uma superfície coberta por vegetação específica rasteira, uniformemente distribuída, em fase de crescimento ativo, em solo com perfeita condutibilidade hídrica e mantido sempre úmido, próximo à capacidade de campo, ou seja, a ETo expressa a demanda evaporativa da atmosfera de um local específico em uma época do ano e não considera as características da cultura e fatores do solo, sendo função dos fatores do tempo.

ETo é, portanto, de fundamental significância para o dimensionamento de sistemas e para o manejo da água de irrigação, o que requer a adoção de estudos, avaliações e ajustes para sua correta utilização. A escolha de um método de estimativa da ETo depende de uma série de fatores, tais como: da disponibilidade de dados meteorológicos e da escala de tempo desejada. Métodos complexos, como o de Penman Monteith, que apesar de ser adotado pela Food and Agriculture Organization (FAO) como o método padrão de estimativa da ETo na escala diária, exigem grande número de variáveis meteorológicas e, por isso, têm aplicação limitada, somente sendo utilizados quando há disponibilidade de todos os dados necessários (Andrade Junior et al., 2003).

Do município de Capim, $\mathrm{PB}$, região que possui grande potencial para o cultivo da cana-de-açúcar, o demonstrativo da evapotranspiração assume elevado conceito; assim, esta região tem estratégica fundamental para o desenvolvimento da agricultura na Paraíba, daí a necessidade de se realizar trabalhos científicos neste local, dentre os quais se destaca a estimativa da evapotranspiração de referência, motivo pelo qual se objetivou estimar a evapotranspiração de referência diária nesse município, pelos métodos Penman-Monteith, Linacre e Radiação, e verificar qual dos dois últimos métodos citados se ajusta e se correlaciona melhor com o sugerido como padrão pela FAO.

\section{MATERIAL E MÉTODOS}

Os dados foram obtidos através da Plataforma de Coleta de Dados (PCD) do Centro de Previsão de Tempo e Estudos Climáticos (CPTEC) e Instituto Nacional de Pesquisas Espaciais (INPE). A PCD está instalada na Fazenda Capim II (6,94 $\left.\mathrm{S} ; 35,12^{\circ} \mathrm{W} ; 103 \mathrm{~m}\right)$ na propriedade da Destilaria Miriri S.A., localizada no município Capim, PB, distando 40 km da Capital João Pessoa, de onde é feito seu principal acesso pela BR 101. Conforme o IBGE (2007), a população do município consiste de 5.207 habitantes e a área territorial de $78 \mathrm{~km}^{2}$. Desta forma, tais valores são: temperatura do ar, umidade relativa do ar, irradiação solar, pressão atmosférica e velocidade do vento, onde o período de coleta dos mesmos abrange de 21 de dezembro de 2006 a 20 de dezembro de 2007.

\section{Método de Penman-Monteith}

No cálculo da ETo, através do método de Penman-Monteith considera-se uma cultura que se assemelha a um gramado de altura uniforme de $0,12 \mathrm{~m}$, regada, crescendo ativamente, sombreando por completo o solo, com albedo de 0,23 e resistência superficial de $70 \mathrm{~s} \mathrm{~m}^{-1}$, pela Eq. 1 (Allen et al., 1998):

$$
\mathrm{EToPM}=\frac{0,408 \Delta\left(\mathrm{R}_{\mathrm{N}}-\mathrm{G}\right)+\mathrm{y} \frac{900}{\mathrm{~T}+273} \mathrm{U}_{2}(\mathrm{es}-\mathrm{e})}{\Delta+\mathrm{y}\left(1+0,34 \mathrm{U}_{2}\right)}
$$

em que:

EToPM - é a evapotranspiração de referência em $\mathrm{mm} \mathrm{d}^{-1}$ $\Delta$ - é a inclinação da curva de saturação do vapor no ponto correspondente à temperatura do ar, $\mathrm{kPa}{ }^{\circ} \mathrm{C}^{-1}$

$\mathrm{Rn}$ - é o saldo de radiação, $\mathrm{MJ} \mathrm{m}^{-2} \mathrm{~d}^{-1}$

$\mathrm{y}$ - é o coeficiente psicrométrico, $\mathrm{kPa}{ }^{\circ} \mathrm{C}^{-1}$

$\mathrm{U}_{2}$ - é a velocidade do vento a $2 \mathrm{~m}$ de altura do solo, $\mathrm{m} \mathrm{s}^{-1}$

$\mathrm{T}$ - é a temperatura do ar, ${ }^{\circ} \mathrm{C}$

es - é a pressão de saturação do vapor em kPa, a pressão real do vapor se apresenta por (e), $\mathrm{kPa}$

$\mathrm{G}$ - é a densidade do fluxo de calor no solo expresso, $\mathrm{MJ} \mathrm{m}^{-2} \mathrm{~d}^{-1}$

\section{Método de Linacre}

O método de Linacre é um método empírico sugerido para a estimativa da evapotranspiração de referência e aplicável por ser simples e de fácil uso, em virtude de requerer apenas dados de temperatura do ar, altitude e latitude do lugar (Linacre, 1977):

$$
\mathrm{EToL}=\frac{700 \frac{\mathrm{T}_{\mathrm{NM}}}{100-\phi}+15\left(\mathrm{~T}-\mathrm{T}_{\mathrm{d}}\right)}{(80-\mathrm{T})}
$$

em que:

EToL - é a evapotranspiração de referência, $\mathrm{mm} \mathrm{d}^{-1}$ $\phi$ - é a latitude em graus

R. Bras. Eng. Agríc. Ambiental, v.14, n.2, p.196-202, 2010. 
$\mathrm{T}_{\mathrm{NM}}$ - é temperatura média do ar a nível do mar, ${ }^{\circ} \mathrm{C}$ $\mathrm{T}_{\mathrm{d}}$ - é a temperatura do ponto de orvalho, ${ }^{\circ} \mathrm{C}$

$\mathrm{T}$ - é a temperatura do ar, ${ }^{\circ} \mathrm{C}$

\section{Método de radiação}

O método de radiação apresentado na Eq. 3 possui o fator de correção (fr) que depende da velocidade do vento e da umidade relativa média do ar.

$$
\mathrm{EToR}=\mathrm{fr}\left(\frac{\Delta}{\Delta+\gamma}\right) \operatorname{Rs}
$$

donde:

EToR - é a evapotranspiração de referência dada em $M J ~ m^{-2} \mathrm{~d}^{-1} \mathrm{e}$ em seguida é convertida para $\mathrm{mm} \mathrm{d}^{-1}$

$\mathrm{R}_{\mathrm{S}}$ - é a radiação solar global incidente na superfície, $\mathrm{MJ} \mathrm{m}^{-2} \mathrm{~d}^{-1}$

$\Delta$ - é a inclinação da curva de saturação do vapor, $\mathrm{kPa}{ }^{\circ} \mathrm{C}^{-1}$

$\gamma$ - é o coeficiente psicrométrico, $\mathrm{kPa}^{\circ} \mathrm{C}^{-1}$

\section{RESULTADOS E DISCUSSÃO}

\section{Verão de 2007}

Calcularam-se as estimativas da evapotranspiração de referência pelos métodos de Penman-Monteith (EToPM), Linacre (EToL) e Radiação (EToR) representadas na Figura 1.

Observa-se que a EToPM e a EToR se ajustam consideravelmente, obtendo-se os menores valores no trigésimo dia do mês de dezembro, ambas em média de 3,0 $\mathrm{mm} \mathrm{d}^{-1}$, em que o valor da radiação solar global acumulada atingiu o menor valor da estação, ou seja, em torno de $12 \mathrm{MJ} \mathrm{m}^{-2} \mathrm{~d}^{-1}$; nesse mesmo dia a precipitação pluvial acumulada obtida foi de $16,5 \mathrm{~mm}$; já no terceiro dia do mês de janeiro a EToR obteve valor de $7,9 \mathrm{~mm} \mathrm{~d}^{-1}$, isto é, o maior valor obtido no verão de 2007, dia em que o valor: da radiação solar global acumulada foi de $28,4 \mathrm{MJ} \mathrm{m}^{-2}$, temperatura máxima de $30,9^{\circ} \mathrm{C}$ e a velocidade do vento $4,9 \mathrm{~m} \mathrm{~s}^{-1}$. A EToL variou praticamente durante toda a estação entre 3,0 e 4,0 $\mathrm{mm} \mathrm{d}^{-1}$, com pequena exceção no décimo sétimo dia do mês de março, quando o valor foi de $4,2 \mathrm{~mm} \mathrm{~d}^{-1}$, pelo fato de que a temperatura nessa data se elevou um pouco mais, em torno de $2{ }^{\circ} \mathrm{C}$ em relação à média constatada na estação.

Da mesma forma, Henrique (2006) notou, em Campina Grande, PB, que no verão de 2004 a estimativa da evapotranspiração de referência através do método de Linacre obteve o pior ajuste durante todos os dias da estação com os métodos de Penman-Monteith e Hargreaves.

Apresenta-se, na Figura 2, a correlação entre a estimativa da evapotranspiração de referência pelos métodos PenmanMonteith (EToPM) e Radiação (EToR).

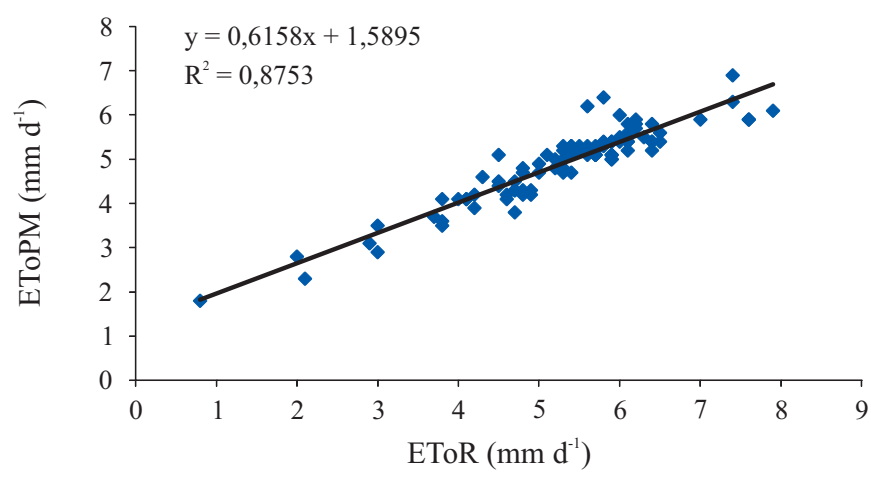

Figura 2. Correlação entre os métodos de Penman-Monteith (EToPM) e o de Radiação (EToR), no verão de 2007 no município de Capim, PB

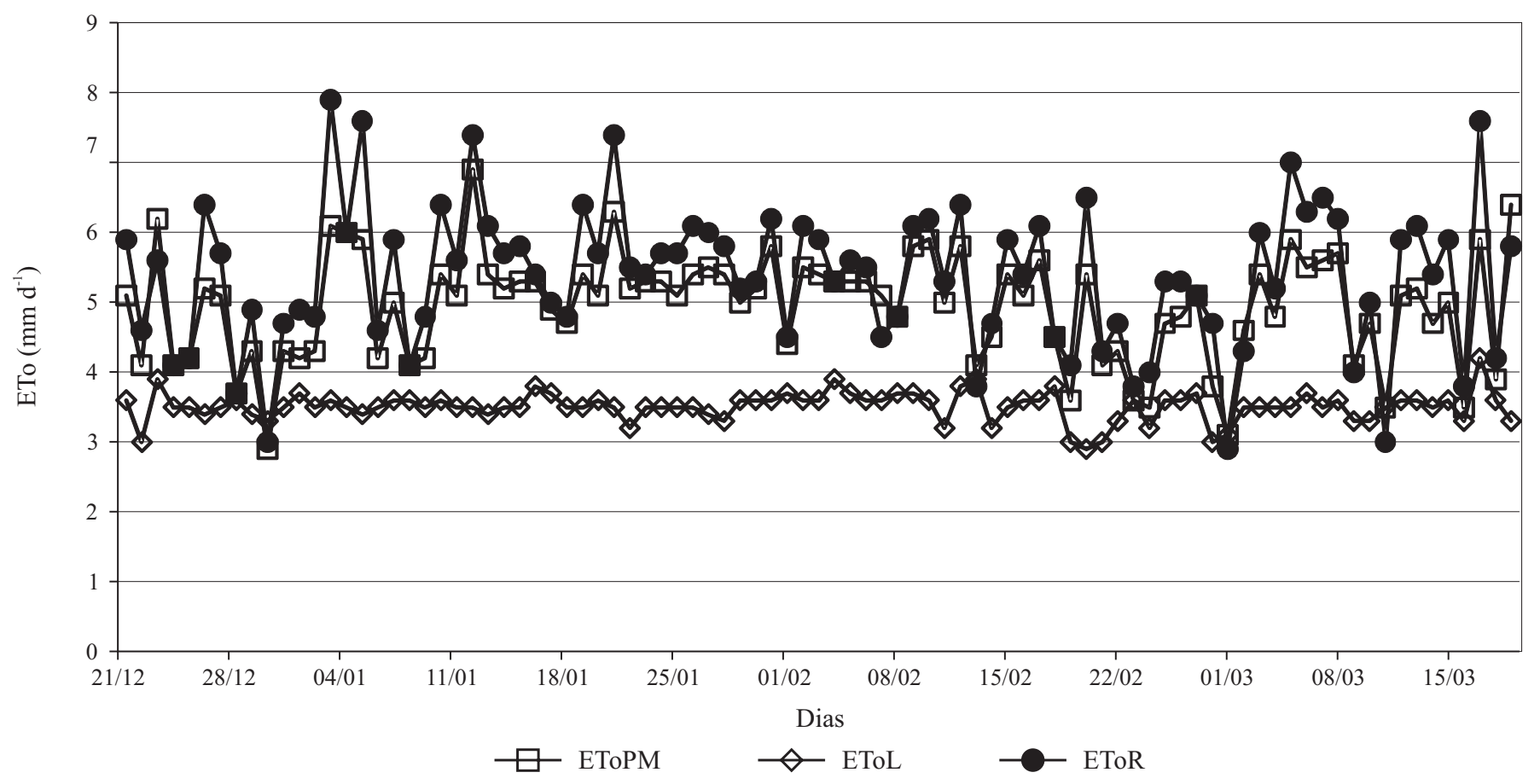

Figura 1. Evapotranspiração de referência (ETo) no verão de 2007 no município de Capim, PB 
A correlação entre EToPM e EToR apresenta-se de forma considerável, com valor de coeficiente de determinação $\left(\mathrm{R}^{2}\right)$ igual a 0,8753 .

Mandelli \& Conceição (2005), compararam valores estimados com as equações de Hargreaves e Samani e outras, com o método Penmam-Monteith; utilizando o coeficiente de desempenho, concluíram que os métodos de estimativa de ETo que empregam a radiação solar incidente, apresentaram desempenhos superiores aos que utilizam apenas valores da temperatura do ar e que, dentre estes, o de Hargreaves e Samani foi o que indicou o melhor desempenho para o município de Bento Gonçalves, RS.

\section{Outono de 2007}

Penman-Monteith (EToPM), Linacre (EToL) e Radiação (EToR) são as estimativas de evapotranspiração de referência representadas na Figura 3.

Constata-se que a linha que representa a EToL não se ajusta às outras ETo, vez que, semelhante ao verão de 2007, se manteve entre 3,0 a 4,0 $\mathrm{mm} \mathrm{d}^{-1}$ com raras exceções; por outro lado, a EToPM e a EToR se ajustam durante toda a estação, observando-se que, do décimo oitavo dia do mês de abril ao vigésimo do mês de junho, as oscilações se tornaram mais acentuadas em virtude, possivelmente, do fato de que, neste período, a radiação solar global acumulada variou em média de $15,5 \mathrm{MJ} \mathrm{m}^{-2} \mathrm{~d}^{-1}$ contra 21,0 $\mathrm{MJ} \mathrm{m}^{-2} \mathrm{~d}^{-1}$ dos primeiros dias do outono de 2007; ressalta-se principalmente o mês de maio, em que esta variável meteorológica oscilou entre 10 e $27 \mathrm{MJ} \mathrm{m}^{-2} \mathrm{~d}^{-1}$; ainda neste mês as chuvas variaram muito de um dia para outro e, por consequência, a nebulosidade na região, salientando que a precipitação acumulada no mês de maio foi de $109 \mathrm{~mm}$.

Também se observa que no décimo quarto dia de junho a EToPM e a EToR atingiram seus maiores valores na esta- ção, haja vista que, entre os décimo terceiro e décimo sexto dias de junho, não ocorreram chuvas embora este mês tenha sido extremamente chuvoso resultando uma precipitação pluvial acumulada de $362 \mathrm{~mm}$; portanto, Reis et al. (2007) verificaram bom ajuste entre a EToPM e a EToR, quando objetivaram a realização do estudo comparativo das equações empíricas para estimativa da evapotranspiração de referência, para as condições climáticas dos municípios de Sooretama, Cachoeiro de Itapemirim e Venda Nova do Imigrante localizados, respectivamente, nas regiões: Norte, Sul e Serrana do Estado do Espírito Santo.

Está representada na Figura 4 a correlação entre a estimativa da evapotranspiração de referência pelos métodos de Penman-Monteith (EToPM) e Radiação (EToR).

O coeficiente de determinação igual a 0,9584 fornece sustentação ao bom ajuste verificado na Figura 4, entre a EToPM e a EToR, tornando-se um pouco mais representativa a correlação nessa estação quanto ao verão de 2007; da mesma forma, Oliveira \& Carvalho (1998) realizaram estudo

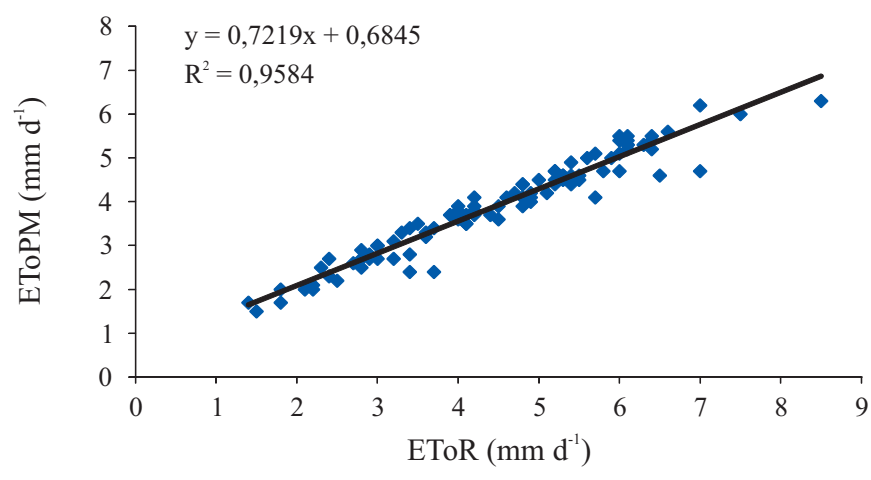

Figura 4. Correlação entre os métodos de Penman-Monteith (EToPM) e o de Radiação (EToR), no outono de 2007 no município de Capim, PB

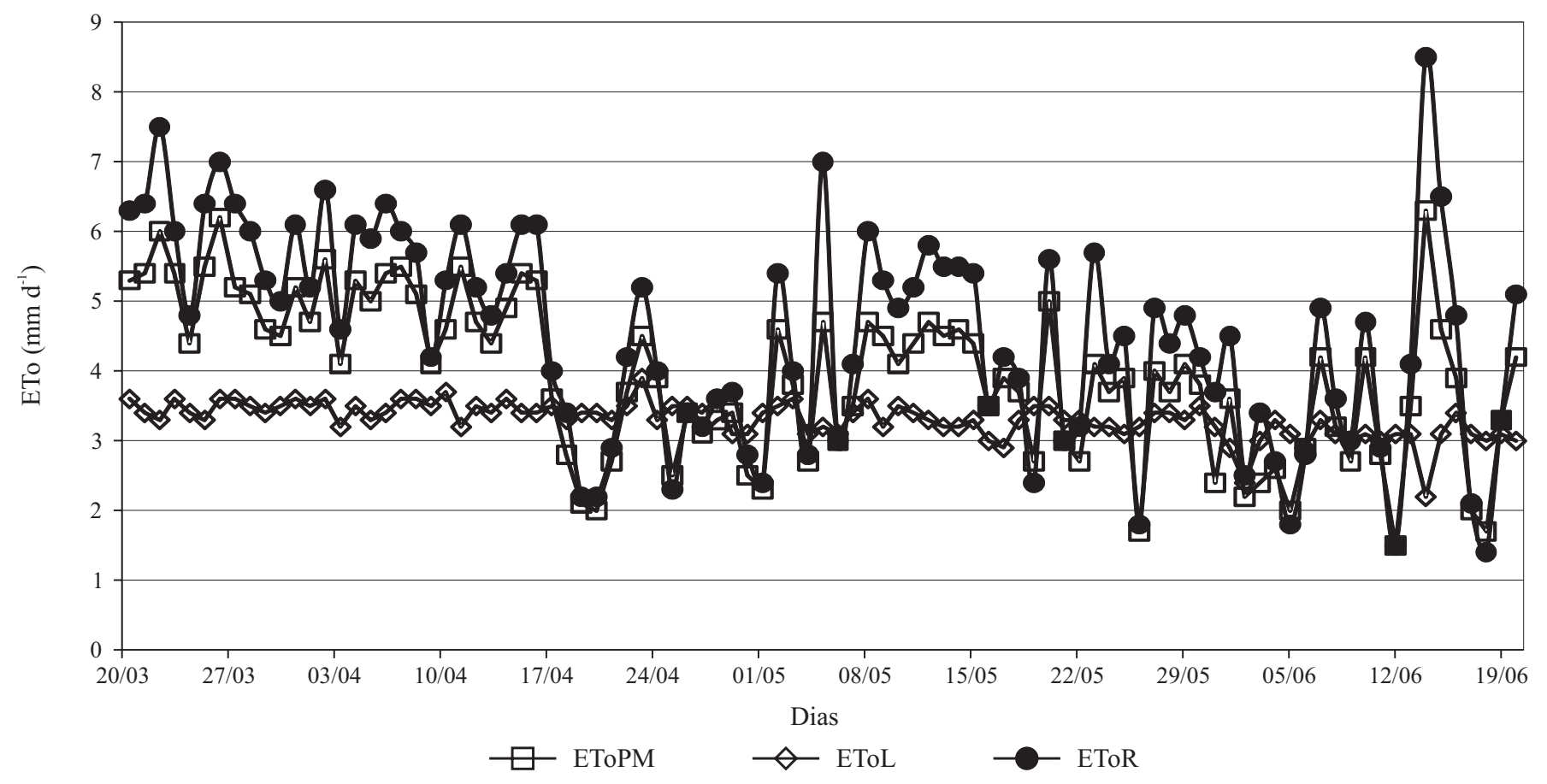

Figura 3. Evapotranspiração de referência (ETo) no outono de 2007 no município de Capim, PB 
com o objetivo de avaliarem a correlação entre diferentes métodos utilizados na estimativa da evapotranspiração de referência nos municípios de Seropédica e Campos, RJ, referente ao método padrão (EToPM), e constataram que a EToR apresentou boa correlação com valores de $\mathrm{R}^{2}$ iguais ou maiores que 0,95 permitindo, assim, a utilização da equação, como forma de estimativa de ETo, nas duas localidades estudadas.

\section{Inverno 2007}

Na Figura 5 se acham as estimativas da evapotranspiração de referência calculadas pelos métodos de Penman-Monteith (EToPM), Linacre (EToL) e Radiação (EToR).

Observa-se, para o inverno de 2007, que os valores diários da evapotranspiração de referência; obtidos pelos métodos de Penman-Monteith e Radiação, tiveram praticamente variações e comportamentos bem semelhantes durante toda a estação, variando ambas em média de 4,0 $\mathrm{mm} \mathrm{d}^{-1}$ com restrição ao vigésimo terceiro dia de agosto em que a EToR atingiu o maior valor; também neste mesmo dia o valor da umidade relativa do ar foi o dos menores do mês, em torno de $74 \%$ e a velocidade média do vento se tornou mais intensa.

No estudo comparativo de modelos de estimativa da evapotranspiração de referência para algumas localidades no estado de Goiás e Distrito Federal, feito por Oliveira et. al. (2001), também se obtiveram bons ajustes entre as EToPM e EToR, em que os autores da pesquisa concluíram que, pelos resultados obtidos para o município de Goiânia, todos os modelos mostraram alta significância com o de Penman-Monteith e o modelo que mais se aproximou do padrão foi o Penman, seguido dos modelos de Hargreaves e Radiação.

A EToL se manteve próxima de $3,0 \mathrm{~mm} \mathrm{~d}^{-1}$ durante toda a estação, destacando-se apenas o décimo nono dia do mês de julho e o décimo de setembro quando, em ambos, os valores obtidos foram em torno de $2,5 \mathrm{~mm} \mathrm{~d}^{-1}$; nesses dias a temperatura máxima do ar se tornou elevada em $3{ }^{\circ} \mathrm{C}$ em relação à média dos outros dias da estação.

A representação da correlação entre a estimativa da evapotranspiração de referência através dos métodos, PenmanMonteith (EToPM) e Radiação (EToR) está na Figura 6.

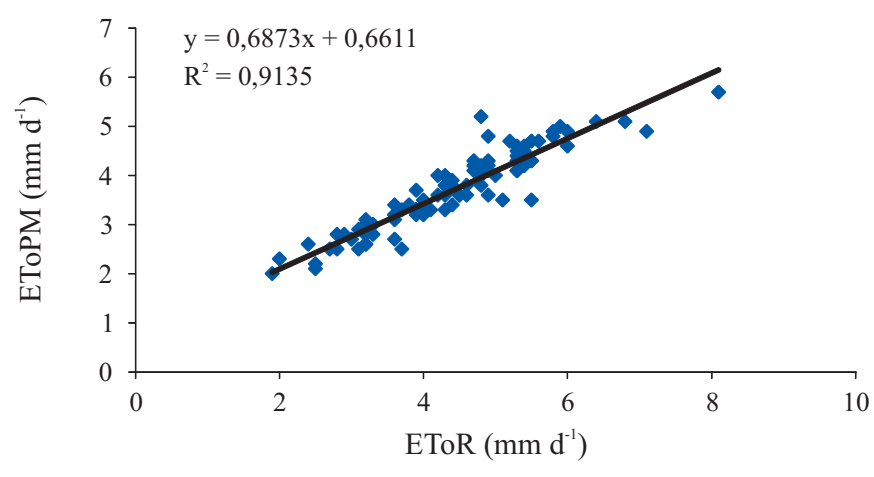

Figura 6. Correlação entre os métodos de Penman-Monteith (EToPM) e o de Radiação (EToR), no inverno de 2007 no município de Capim, PB

Para o inverno de 2007 o coeficiente de determinação permanece com valor bem significativo, praticamente o mesmo do outono do ano referenciado na Figura 4.

\section{Primavera 2007}

As estimativas da evapotranspiração de referência foram calculadas pelos métodos de Penman-Monteith (EToPM), Linacre (EToL) e Radiação (EToR) estão representadas na Figura 7.

A ETo de Penman-Monteith e a ETo de Radiação se

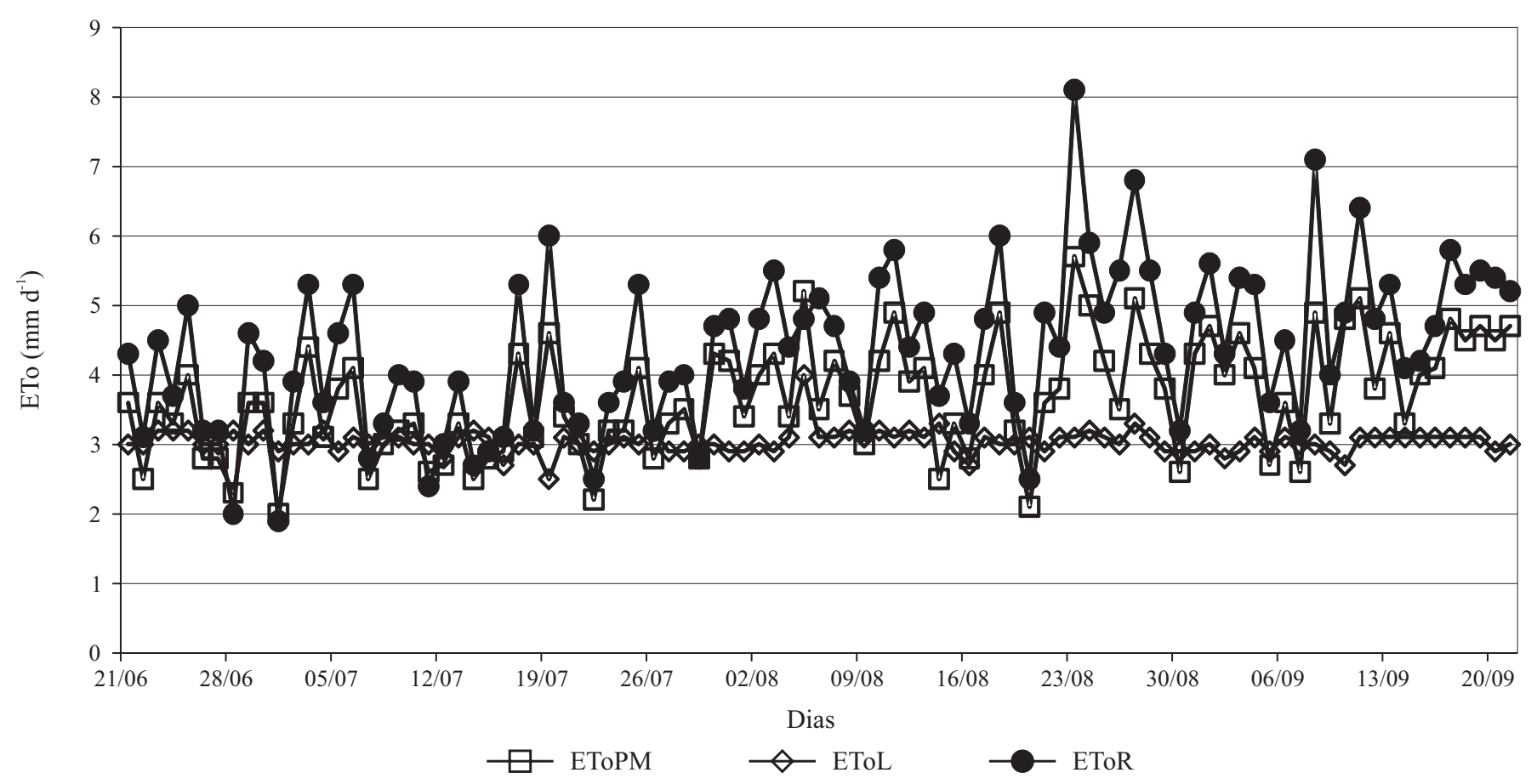

Figura 5. Evapotranspiração de referência (ETo) no inverno de 2007 no município de Capim, PB 


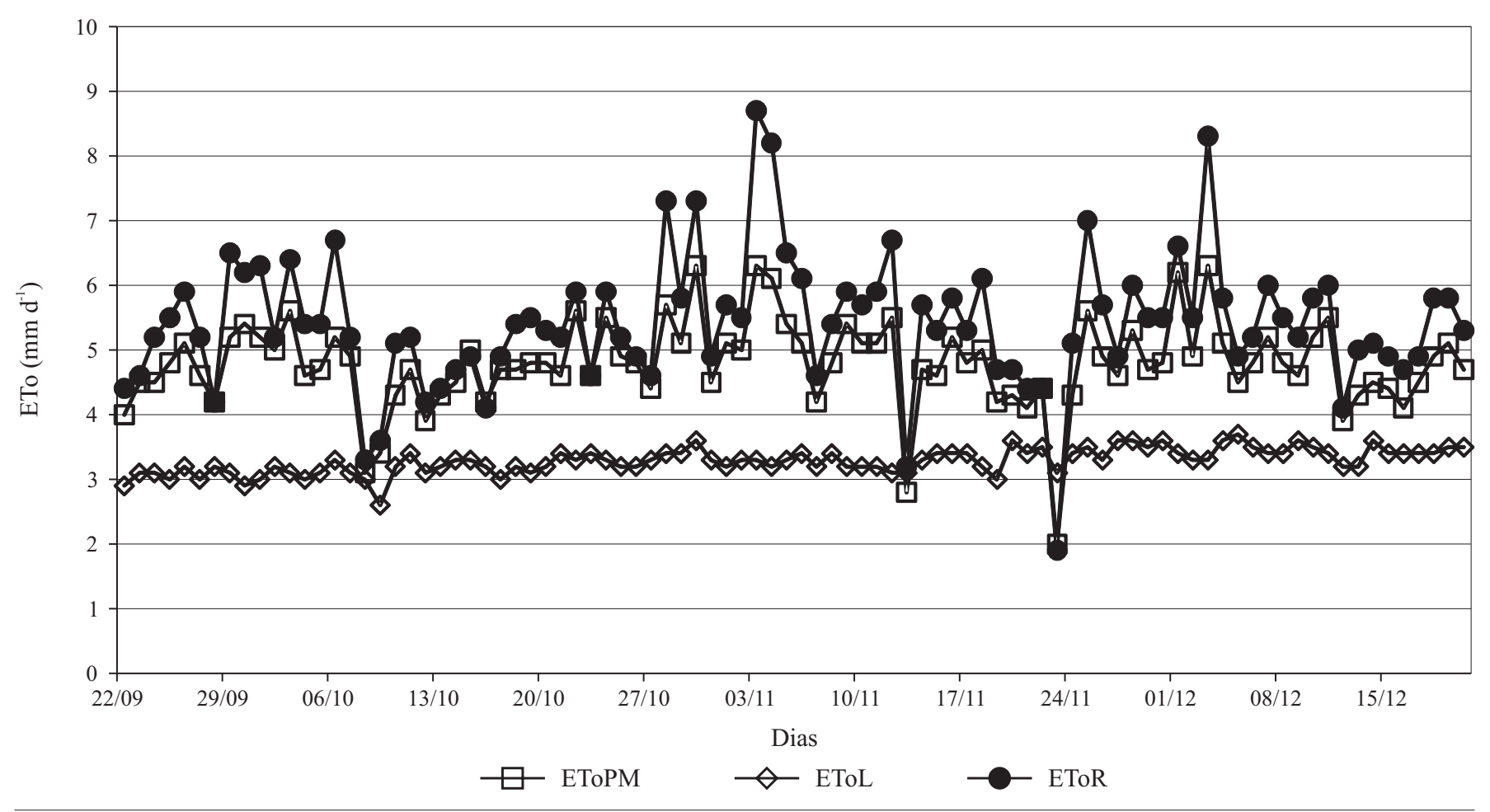

Figura 7. Evapotranspiração de referência (ETo) na primavera de 2007 no município de Capim, PB

ajustaram muito bem durante toda a estação da primavera de 2007, salientando-se que os menores valores obtidos em ambas ocorreram no vigésimo terceiro dia de novembro, quando a umidade relativa do ar atingiu o maior valor do mês, em volta de $90 \%$, com o menor valor obtido na radiação solar global acumulada em $8,5 \mathrm{MJ} \mathrm{m}^{-2}$ com precipitação pluvial acumulada no dia de $17 \mathrm{~mm}$; já os maiores valores obtidos na EToPM e EToR aconteceram no terceiro dia dos meses de novembro e dezembro, dias em que o acúmulo de radiação solar global foi o maior no mês e a umidade relativa do ar se tornou uma das menores, em média de $75 \%$.

Observa-se, então, que não só nessa estação mas em todas as outras estações do ano 2007, no município de Capim, a EToPM e EToR se ajustaram muito bem. Oliveira et al. (2008) ao avaliarem várias estimativas de evapotranspiração de referência em Gameleira, PE, utilizando lisímetro de pesagem hidráulica, evidenciaram que a ETo estimada pelo método da Radiação foi a que mais se ajustou aos valores obtidos no lisímetro.

Constata-se também que a EToL se apresenta de forma um pouco mais harmônica variando em $0,7 \mathrm{~mm} \mathrm{~d}^{-1}$ na citada estação, em volta da faixa de $3,3 \mathrm{~mm} \mathrm{~d}^{-1}$; ressalta-se que a temperatura do ar na região varia sempre em $2{ }^{\circ} \mathrm{C}$ e, quanto a Campina Grande, PB, no inverno de 2004 a temperatura do ar variou de 20,2 a $24,3^{\circ} \mathrm{C}$ (Henrique, 2006). Por outro lado, Mendonça et al. (2003) que fizeram análises de várias estimativas de evapotranspiração de referência em Campos dos Goytacazes na região norte-fluminense no Rio de Janeiro, constataram que a média diária no mês de fevereiro de 2000 para o método de Linacre, foi de $4,5 \mathrm{~mm} \mathrm{~d}^{-1}$.

Na Figura 8, a seguir, se encontra a correlação entre a estimativa da evapotranspiração de referência através dos métodos de Penman-Monteith (EToPM) e Radiação (EToR).

Comprova-se, pela Figura 8, a correlação bem representativa entre as EToPM e EToR na primavera de 2007, cujo coeficiente de determinação de valor igual a 0,8807 , justifica a correlação.

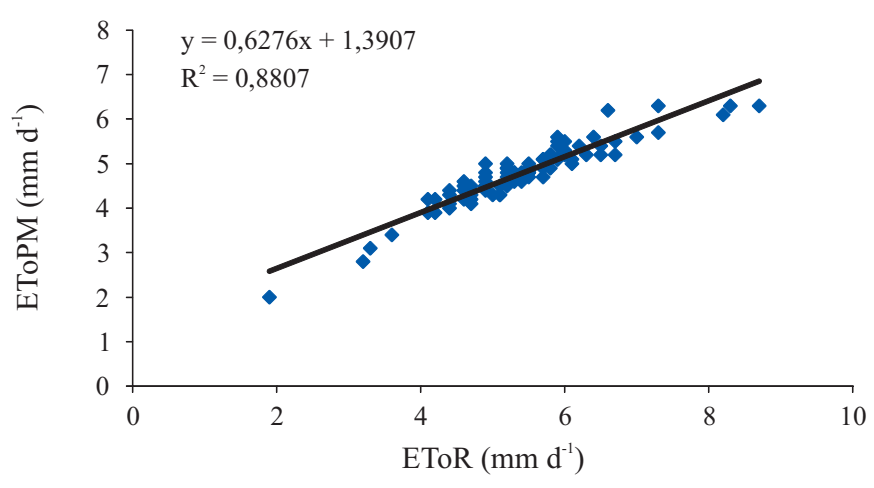

Figura 8. Correlação entre os métodos de Penman-Monteith (EToPM) e o de Radiação (EToR), na primavera de 2007 no município de Capim, PB

\section{CONCLUSÕES}

1. A estimativa da evapotranspiração de referência, obtida pelo método de Radiação no município de Capim, PB, ajustou-se e se correlacionou muito bem a todas as estações de ano com o método de Penman-Monteith, sugerido pela FAO.

2. O método de Linacre, para estimar a evapotranspiração de referência, não se ajustou com nenhum dos métodos analisados na pesquisa. 


\section{LITERATURA CITADA}

Allen, R. G.; Pereira, L. S.; Raes, D. Crop evapotranspiration. Guidelines for computing crop water requirements. Rome: FAO, 1998. p.300. Irrigation And Drainage Paper 56

Andrade Junior, A. S. de; Bastos, E. A.; Sentelhas, P. C.; Silva, A. A. G. da. Métodos de estimativa da evapotranspiração de referência diária para Parnaíba e Teresina, Piauí. Revista Brasileira de Agrometeorologia, v.11, n.1, p.63-68, 2003.

Henrique, F. de A. N. Estimativa da evapotranspiração de referência em Campina Grande. Campina Grande: UFCG, 2006. 107p. Dissertação Mestrado

IBGE - Instituto Brasileiro de Geografia e Estatística. Contagem da população em 2007, publicado no Diário Oficial da União de 05/10/2007. <http://www.ibge.gov.br/home/estatistica/populacao/contagem2007/PB.pdf>. 20 Fev. 2008.

Linacre, E. T. A simple formula for estimating evapotranspiration rates in various climates, using temperature data alone. Agricultural Meteorology, v.18, n.1, p.409-424, 1977.

Mandelli, F.; Conceição, M. A. F. Comparação entre métodos de estimativa da evapotranspiração de referência em Bento Gonçalves, RS. Revista Brasileira Agrometeorologia, v.13, n.2, p.303-307, 2005.

Mendonça, J. C.; Sousa, E. F. de; Bernardo, S.; Dias, G. P.; Grippa, S. Comparação entre métodos de estimativa da evapotranspiração de referência (ETo) na região Norte Fluminense, RJ. Revista Brasileira de Engenharia Agrícola e Ambiental, v.7, n.2, p.275-279, 2003.
Oliveira, L. F. C. de; Carvalho, D. F. de; Romão, P. A.; Cortês, F. C. Estudo comparativo de modelos de estimativa da evapotranspiração de referência para algumas localidades no Estudo de Goiás e Distrito Federal. Pesquisa Agropecuária Tropical, v.31, n.2, p.121-126, 2001.

Oliveira, L. M. M. de; Montenegro, S. M. G. L.; Azevedo, J. R. G. de; Santos, F. X. Evapotranspiração de referência na bacia experimental do riacho Gameleira, PE, utilizando-se lisímetro e métodos indiretos. Revista Brasileira de Ciências Agrárias, v.3, n.1, p.58-67, 2008.

Oliveira, M. A. A. de; Carvalho, D. F. de. Estimativa da evapotranspiração de referência e da demanda suplementar de irrigação para o milho em Seropédica e Campos, Estado do Rio de Janeiro. Revista Brasileira de Engenharia Agrícola e Ambiental, v.2, n.2, p.132-135, 1998.

Pozzebon, E. J.; Cunha, P.; Cavalcanti, A. C.; Silva, L. M. C. Procedimentos para pedidos de outorga de direito de uso da água para irrigação. In: Workshop sobre Água, Agricultura e Meio Ambiente no Estado de São Paulo, 2003. Anais... Jaguariúna: Embrapa Meio Ambiente, 2003. CD Rom

Reis, E. F. dos; Bragança, R.; Garcia, G. de O.; Pezzopane, M.; Tagliaferre, C. Estudo comparativo da estimativa da evapotranspiração de referência para três localidades do Estado do Espírito Santo no período seco. Idesia, v.25, n.3, p.75-84, 2007.

Turco, J. E. P.; Faria, M. T.; Fernandes, E. J. Influência da forma de obtenção do saldo de radiação na comparação de métodos de estimativa da evapotranspiração de referência. Irriga, v.10, n.3, p.215-228, 2005. 